\title{
Dental management of COPD patient
}

\author{
SS Rahman1, M Faruque2, MHA Khan3, SA Hossain4
}

\begin{abstract}
Summary
Chronic obstructive pulmonary diseases have increased in prevalence and the rate of death of this chronic inflammatory disease of the airways has also risen despite recent advances in medical treatments. The dental health care professionals must be prepared to treat more medically compromised individuals. Because dental professionals operate at the origin of the upper airway and many dental procedures are deemed stressful, patients with chronic respiratory diseases are at special risk. This article will review the patho-physiology and discuss the recognition and management of dental patients with these diseases and provide an understanding on how to avoid precipitating factors that could initiate an acute episode in the dental care setting. The most important factor in preventing COPD is helping patients stop smoking.
\end{abstract}

Bang Med J (Khulna) 2011; 44 : 21-24

\section{Introduction}

The Management of patients with Chronic Obstructive Pulmonary Disease (COPD) may pose a challenge to the dental surgeon. A thorough understanding of the COPD diseases is paramount to the successful treatment of these individuals. Morbidity and mortality for COPD patients have increased over time. These varieties of patient present particular problem both in diagnosis and treatment planning and thereby dental therapeutics may need a modification.1 The American thoracic society has defined COPD as a disease state characterized by the presence of air flow obstruction either due to chronic bronchitis or emphysema, the airflow obstruction is generally progressive may be accompanied by airway hyperactivity and may be partially reversible.2 COPD attacks the air ways and air sacs within the lungs, which make breathing difficult. As destructive changes progress, lung mechanism are altered due to the disease, increasing the work of breathing by 10 to 20 times of a normal person.3 COPD afflicts about 15 million Americans.4 COPD accounts for about 110,000 deaths/ year and 95000 new patients are diagnosed annually. An average of 9.8 years of life are lost in patients with COPD.5 COPD is also a significant cause of disability that becomes progressively worse if nothing is done to control it.6 In addition to the definition of American Thoracic society, British Thoracic society adds reduced forced expiratory volume in 1 second (FEVI) and reduced FEVI/FVC ratio which does not change markedly over several months.7 Now a days increasing number of COPD patients are coming to health care management centre for dental treatment. But due to lack of knowledge of dental management protocol for COPD patient, we refer the patient to tertiary level hospital. For this reason most of the COPD patients do not get any dental treatment specially economically deprived groups.

\section{Aetiology}

Most cases of COPD occur as a result of long term exposure to lung irritants that damage the lungs and the airways. The primary cause of COPD is cigarette smoking. Smoking attributes to $87 \%$ to $91 \%$ of COPD development. Other contributory factors to COPD include recurrent respiratory tract infection, air pollution, cotton textile dust heredity and aging.8 Survey reveals $15 \%$ of the smoker likely to develop clinically significant COPD and the risk of COPD is 8.8 times higher in males smoker and 5.9\% higher in female smoker compared with non smoker of the same gender.9 It is proposed that cigarette smoke can activate macrophages in the lung to release factors that directly stimulate neutrophils to secrete elastases and combine with oxidants and free radicals, which in turn oxidize a1-anti trypsin.10 It should be noted, however, that very, few patients with chronic obstructive pulmonary disease exhibit symptoms exclusive of chronic bronchitis or emphysema. Infact, many patients share symptoms attributed to both conditions.11

Both conditions share a common etiology, with exposure to tobacco smoke being the primary cause of chronic obstructive pulmonary disease. However, for reasons that are unclear, only about 15 percent of cigarette smokers actually develop clinically significant COPD, while tobacco smoking is known to account for 80 percent of the risk acquiring disease.12 With smoking cessation in individuals with mild to moderate airflow obstruction, the rate of deterioration can return to that seen in nonsmokers, and their symptoms may decrease.13 Children of smokers are known to have a higher incidence of respiratory symptoms and respiratory function is decreased when measured through pulmonary function test.14 Breathing in secondhand smoke, air pollution and chemical fumes or dust from the environment or work place also can contribute to COPD. Second hand smoke is smoke in the air from other people smoking.

Environmental pollution such as air pollution, indoor irritant sources and occupational air borne hazard. Cities like Dhaka, Kolkata, Delhi having in higher amount of SPM (Suspended Particulate Matter) and RSMP (Respiratory Suspended Particulate Matter) posses of greater threat to its inhabitants of developing COPD.

In rare cases, a genetic condition called alpha-I antitrypsin deficiency may play a role in causing COPD. People who have this condition have low levels of alpha-I antitrypsin (AAT) protein made in the liver. Having a low level of the AAT protein

1. Sheikh Shahidur Rahman MPH, Assistant Professor, Dept. of Dental Surgery, Khulna Medical College, Khulna.

2. Mohammad Faruque MMED, Director, (Dental), DGHS, Dhaka.

3. Md. Haider Ali Khan MPH, Assistant Professor, Dept. of Dental Public Health, Dhaka Dental College, Dhaka

4. Shaikh Amir Hossain FCPS, Assistant Professor, Dept. of Medicine, Khulna Medical College. 
can lead to lung damage and COPD if exposed to smoke or other lung irritants. If anyone has this condition and smoke, COPD can worsen very quickly.

\section{Clinical presentation}

The signs and symptoms of COPD include

- An ongoing cough or a cough that produces large amounts of mucus (often called smokers cough)

- Shortness of breath, especially with physical activity.

- Wheezing (a whistling or squeaky sound when you breathe)

- Chest tightness.

However, not everyone who has these symptoms has COPD. Some of the symptoms of COPD are similar to the symptoms of other diseases and conditions. If COPD is severe and develops corpulmonale results right sided cardiac failure leads to swelling in ankles, feet, legs, a bluish' colour on lips due to a low blood oxygen level and shortness of breath.

COPD symptoms usually slowly worsen overtime. At first, if symptom is mild, may not notice, or may adjust lifestyle to make breathing easier. For example, anyone may take the elevator instead of the stairs. Severity of symptoms are depends on how much lung damage. If patient keep smoking, the damage will occur faster than if stop smoking.

Some severe symptoms may require treatment in a hospital. If patient with the help of family members or friends, are unable - should seek emergency care if

- Having a hard time catching breath or talking Lips or fingernails turn blue or gray

- Not mentally alert

- Heart beat is very fast

- The recommended treatment for symptoms that are getting worse is not working.

Patients with suspected history of the disease or with known disease status should be evaluated and should undergo some routine investigation. These are

i) Thorough clinical history including risk factor

ii) Lung function test

iii) Spirometry/Lung volume studies

iv) Chest X-ray or chest CT scan

v) An arterial blood gas test

Patient with COPD may have other debilitating medical condition like hypertension, myocardial infarction, can complicate and make the prognosis worst and thus should be carefully investigated along with medical complaints. 15

\section{Dental management protocol}

The primary objective for the dental surgeon in the management of a patient with a medical condition is to prevent any complications related to that condition as a result of dental treatment. The COPD patient can be treated for his or her dental needs when the dental health practitioner has developed a risk assessment that is individualized for the patient. This assessment begins with an appropriate understanding of the patient's medical history. The health history questionnaire and a comprehensive interview by the dental surgeon is the foundation of the risk assessment process. The reduction of stress and avoidance of any procedures that may depress a patient's respiratory function are essential in the management of patients with moderate to severe chronic obstructive pulmonary disease.

Because COPD cannot be cured, medical treatment is directed at reducing the degenerative effects of the disease and managing the acute and chronic symptoms of chronic bronchitis or emphysema. Smoking cessation is the single most important therapy for patients with obstructive airway disease and proves to be the greatest challenge for the patient and the physician in managing the disease.16 The smokers should give advice to stop smoking six weeks before dental procedure. Patients offered a professional and reassuring environment with short, focused dental treatments early in the day, placing a patient in a reclined position or the use of rubber dam can contribute to a severe respiratory compromise. Low-flow supplemental oxygen administration via nasal canula at rates of 2 to $4 \mathrm{~L} /$ minute is appropriate even in patients with severe disease. Oxyen theapy is an important therapeutic agent for hypoxemic patients. It can improve survival in COPD patients when uses continuously.17

As a group, patients with asthma, bronchitis and chronic restrictive or obstructive pulmonary disease are best managed with local anaesthesia for out patient procedures.18 The selection of local anesthetic is important when treating COPD. Many local anaesthetic solutions contain sulfites which precipitate acute asthmatic attacks and allergic reactions.19 These compounds are found in local anaesthetic preparations containing epinephrine and levonordefin and the preparations should not be used. If the patient is treated with local anaesthesia the bronchodilator inhaler should be kept ready for use in case of emergency. If there is an acute attack on the table patients can use nebuliser with bronchodilator like salbutamol. From the surgeons point of view the most important aspect is the patient's respiratory reserve and his ability to tolerate general anaesthesia. Outpatient general anaesthesia is not recommended for most patients with COPD.20

Sedation can be considered, but potent sedatives, barbiturates or narcotics should be avoided as they can depress the respiratory drive. Nitrous oxide and high flow rates of oxygen are contraindicated because their use can result in respiratory depression. Anticholinergic or antihistamines can alter tracheobronchial secretion leading to air flow disturbarssesshbiben the symptoms associated with chronic obstructive pulmonary disease are continuous or more severe and their management with the bronchodilators is suboptimal, the use of sustained release theophylline is considered. Theophylline has been shown to improve respiratory muscle function, stimulate the respiratory centre and improve mucocilliary clearance.21 Patients with coexisting cardiac disease, corpulmonale and pulmonary hypertension may benefit from the improved cardiac output, reduced pulmonary vascular resistance and improved myocardium perfusion that theophylline can produce.

When antibiotic therapy is indicated, patients taking theophylline should not be given macrolides (i.e. erythromycin, azithromycin and clarithromycin) ciprofloxin, clindamicin, which can lead IV methylxanthine toxicity. In cases of using NSAIDS, at antiinflammatory doses respiration is stimulated by peripheral and central action. Salicylate causes respiratory depression.

As acetaminophen and Cox-2 inhibitors do not precipitate bronchospasm, it can be used for these patients. The use of corticosteroids has limited applications in the treatment of COPD given that the disease process is primarily one of tissue degeneration and destruction with little or no reversible component.22 The COPD patient is at risk for developing excess cough and mucous production, specially if chronic bronchitis is present. The use of humidified warm air and maintenance of 
adequate hydration is needed to reduce the potential for respiratory depression. 23

Patients with COPD often have a faster metabolism due to increased work of breathing. A high intake of carbohydrate increases C02 production, increasing dyspnea. In light of this problems small frequent high-protein, high-fat meals are indicated for COPD patients. The use of enteral nutrition may be indicated to provide adequate nutritional support after surgery.24

\section{Conclusion}

The dental surgeon in practice today must be prepared to provide care for patients with complex medical conditions. Routine dental care can be provided when the dental health care professionals are knowledgeable about pulmonary diseases and is familiar in the signs, symptoms and management of an emergent episode associated with COPD. Patients with moderate to severe pulmonary disease require a risk assessment for safe and appropriate care.

\section{Reference}

1. Sandip B, Samiron G, Sudip C, Amit R, Saha T K. Dental management for COPD patient. JIDA. 2008; 24 : 18-20.

2. Haslett C, Chilvers ER, Corris P.A. Respiratory disease IN: Haslett christoper, Chilvers Edwin R, Boon Nicholas A, Colledge Nicki R. Davidson's principles and practice of medicine I9th ed. Churchill living stone: Edinburgh; 2002 : 508-513.

3. Collad H, Saint S, Mallhay M. Prevention of ventricular associated pneumonian evidence based systemic review. Ann Intern Med $138: 2003$.

4. Wenzle R. Prevention and control of Nososcomial infections. Fourth edition. Baltimore j Lippincott; William and Wilkens, 2003

5. Cook D et al. Toward understanding evidence uptake: semirecumbency for pneumonia prevention. Critical Care Medicine 2002; 30.

6. National Centre for Health Statistics: Birth and Deaths, United States, Monthly vital statistics report 1997; $46: 5$.

7. Hayes peter C, Mackay Thomas W, Forrest Ewan H, Fisken Roger A. Churchill's Pocket book of medicine. 3rd ed. Harcourt (India) private limited: New Delhi; 2002 : 72-74.
8. Munro CL, Grap MJ. Oral health care in the intensive care unit: State of the science. Am j Crit Care 2004; 13(1).

9. Little James W, Falace Donald A, Miller Craig S, Rhodus Nelson L. Dental management of the medically compromised patient. 6th ed. Mosby: st Louis; 2002 : 125-130..

10. Gapek JE, Pacht ER. Pathogenesis of hereditary emphysema and replacement therapy for a antitrypsin deficiency insight into the more common forms of emphysema chest 1996; $110: 2585$.

11. American Thoracic Sociey. Standards for the diagnosis and care of patients wih chronic obstructive pulmonary disease. Am J Respir Care Med 152: 1995 : 77-121.

12. Sherril DL, Lebowitz M, Burrows B. Epidemiology of chronic obstructive pulmonary disease. Clin Chest Med 1990; 11 : 375-88.

13. RM, Novotny TE. The epidemiology of cigarette smoking and its impact on chronic obstructive pulmonary disease. Am REV respire Dis 140:1990. : 582-4.

14. Brown CA, Crombic IK, Smith WCS. The impact of quality smoking on symptoms of chronic bronchitis: result of the Scottish heart healthy study. Thorax 1991; 46 : 112-4.

15. Foley NM. Chronic obstructive pulmonary disease. SAAD Dig 17(3): $2000: 3-12$.

16. Laskin DM. Oral and Maxillofacial Surgery. Mosby : St Louis; 2002.

17. Dent MR. Hospital - acquired pneumonia: the gift that keeps in taking. Nursing 2004; 34(2).

18. Malik NA. Textbook of Oral and Maxillofacial Surgery. 1st ed, Jaypee: New Delhi; 2005.

19. Cuestaa - Herranz J, et al. Allergic reactions caused by local anaesthetic agents belonging to amide

20. Zimet 1. Phamacological therapy of obstructive airway disease. Clin Chest Med 1990; 11 : 461-86.

21. Mckay SE, Howie ALL et al. Value of theophylline in patients handicapped by COPD. Thorax 1993; $48: 227-32$.

22. Keating VM, Jatakanon A, et al. Effects of inhaled and oral glucocorticoids on inflammatory indices in asthma and COPD. Am J Repair crit care Med 155: 1997 : 542-8

23. Hanowell LH, Junod FL. Pulmonay care of the surgical patient.Mt. Kisco, NY; Futura, 1994.

24. Harrison LB, Sessions RB, Hong WK. Head and Neck Cancer: A Multidisciplinary Approach. Philadelphia: Lippincott - Raven; 1999. 